\title{
Special issue: Environmental photocatalysis
}

As the rapid development of industrial economy, massive consumption of fossil energy leads to increasingly serious environmental pollution. Thus, it is highly obliged to develop green, efficient and robust technologies for fuel production and pollution remediation. Semiconductor-based photocatalysis could realize the production of fuel chemicals, selective transformation/degradation of organic pollutants and even inactivation of microorganisms by using the solar-to-chemical energy conversion hence possesses scientific significance and great application potential. Besides, multidisciplinarily involving semiconductor physics, catalysis and surface science, photocatalysis has become one of the most attractive and pioneering research fields all over the world.

The key to determine the photocatalytic efficiency lays on the highly efficient and robust photocatalysts. It is well known that the light absorption range, photogenerated charge separation and surface catalytic process are three main factors affecting photocatalytic activity. Accordingly, current design of photocatalyst materials focuses on the following aspects: to extend the spectral response to the visible/infrared range by developing novel organic semiconductors; to rationally fabricate covalent/non-covalent heterojunctions aiming at enhanced photogenerated charge separation; to introduce highly active catalytic centers on the photocatalyst surface to facilitate the catalytic reactions; to simultaneously emphasize the application of in-situ techniques and theoretical calculation to uncover the relationship between the structure of photocatalyst and reactivity in depth. All aspects above compose the main research content in photocatalysis.

Centering at the topic of environmental photocatalysis, the research groups with abundant experience and great academic influence have been invited to publish twenty three academic papers in this special issue. The content covers current experimental and theoretical progress on the design and synthesis of environmental photocatalysts as well as the mechanism of photogenerated charge separation and photocatalytic process. We hope this special issue would enable the readers to acquire an in-depth and comprehensive understanding on the progress, opportunity and challenge in environmental photocatalysis. As researchers, we are bound to maintain the beginner's mind, undertake the mission and seize the moment without pity, devoting to develop inexhaustible energy source for forever clear water and blue sky.

Finally, we would like to express our sincere thanks to invited authors, peer reviewers and editorial staffs for their contribution and efforts!

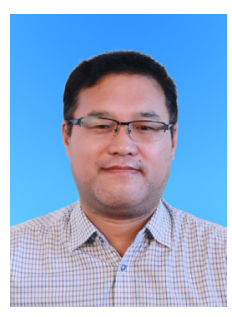

Prof. Liqiang Jing (Guest Editor)

Key Laboratory of Functional Inorganic Materials Chemistry, Ministry of Education, School of Chemistry and Materials Science, Heilongjiang University

E-mail: Jinglq@hlju.edu.cn

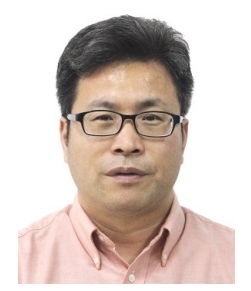

Prof. Chuncheng Chen (Guest Editor)

Key Laboratory of Photochemistry, Institute of Chemistry, Chinese Academy of Sciences

E-mail: ccchen@iccas.ac.cn

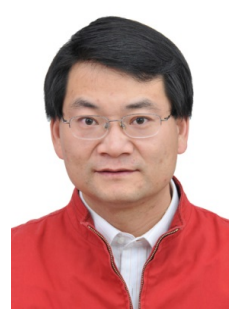

Prof. Taicheng An (Guest Editor)

School of Environmental Science and Engineering, Institute of Environmental Health and Pollution Control, Guangdong University of Technology E-mail: antc99@gdut.edu.cn

DOI: 10.1016/S1872-2067(20)63645-2 | http://www.sciencedirect.com/science/journal/18722067 | Chin. J. Catal., Vol. 41, No. 10, October 2020 (C) 2020, Dalian Institute of Chemical Physics, Chinese Academy of Sciences. Published by Elsevier B.V. All rights reserved. 